\title{
Courant-Snyder formalism of longitudinal dynamics
}

\author{
X. J. Deng $\odot,{ }^{1, *}$ A. W. Chao, ${ }^{2,3}$ W. H. Huang, ${ }^{1}$ and C. X. Tang ${ }^{1}$ \\ ${ }^{1}$ Department of Engineering Physics, Tsinghua University, Beijing 100084, China \\ ${ }^{2}$ Institute for Advanced Study, Tsinghua University, Beijing 100084, China \\ ${ }^{3}$ Stanford University, Stanford, California 94309, USA
}

(Received 27 June 2021; accepted 26 August 2021; published 24 September 2021)

\begin{abstract}
Originating from the stochastic nature of the photon emission process, the diffusion of the electron longitudinal coordinate exists even if the global phase slippage of a storage ring is zero, as we cannot zero all the local phase slippages simultaneously. This quantum diffusion is viewed as the most fundamental limit of the lowest bunch length realizable in an electron storage ring from the single-particle dynamics perspective. Here, we present an analysis of this effect using the Courant-Snyder parametrization in the longitudinal dimension. Analytical formulas for the longitudinal emittance, energy spread, and bunch length are derived. The same formalism is used to discuss the application of multiple radio frequency systems for longitudinal strong focusing. The presented work is expected to be useful in the development of novel light source mechanisms like steady-state microbunching, where a short bunch length or small longitudinal emittance is needed.
\end{abstract}

DOI: 10.1103/PhysRevAccelBeams.24.094001

\section{INTRODUCTION}

Short bunches and small longitudinal emittance are desired by synchrotron light source and lepton collider communities for coherent radiation generation and luminosity increase. One successful method of realizing short bunches in an electron storage ring is implementing a quasi-isochronous (low-alpha) lattice. The reason is the well-known $\sqrt{|\eta|}$ scaling law of the "zero-current" bunch length given by Sands [1], in which $\eta=\alpha-\frac{1}{\gamma^{2}}$ is the phase slippage factor and $\alpha=\frac{1}{C_{0}} \oint \frac{D_{x}(s)}{\rho(s)} d s$ is the momentum compaction factor of the ring, with $\gamma$ the relativistic factor, $C_{0}$ the circumference of the ring, $D_{x}$ the horizontal dispersion, and $\rho$ the bending radius. However, from the single-particle dynamics perspective, there is a fundamental effect limiting the lowest bunch length realizable in an electron storage ring originating from the stochasticity of photon emission time, which was first originally analyzed by Shoji et al. [2,3]. This stochasticity results in a diffusion of the electron longitudinal coordinate $z$ even if the global phase slippage of the ring is zero, as we cannot make all the local or partial phase slippages zero simultaneously. Therefore, there exists a bunch length limit, and the energy spread grows significantly when the bunch length is pushed

\footnotetext{
*dxj11@tsinghua.org.cn
}

Published by the American Physical Society under the terms of the Creative Commons Attribution 4.0 International license. Further distribution of this work must maintain attribution to the author(s) and the published article's title, journal citation, and DOI. close to the limit. This quantum diffusion is of vital importance for new light source concepts like steady-state microbunching (SSMB) [4-13], which is now under active study, and other ideas invoking ultrashort electron bunches in storage rings.

While the treatment of this quantum excitation is intrinsically embedded in Chao's solution by linear matrices (SLIM) formalism [14], which invokes $6 \times 6$ general transport matrices and applies to a $3 \mathrm{D}$ general coupled lattice without the assumption of longitudinal weak focusing, analytical formulas for this effect are still highly desired. The motivation is that these analytical results can help us to better understand its physics and the method to control this quantum diffusion to minimize the longitudinal emittance and bunch length, for example, in an SSMB storage ring. In this paper, we apply the longitudinal Courant-Snyder formalism in a planar uncoupled ring for the analysis of this effect, which can be viewed as an application of the SLIM formalism where a concise analytical result can be obtained. Formulas for the equilibrium longitudinal emittance, energy spread, and bunch length are derived, based on which some important points of this effect are analyzed. The application of multiple radio frequency (rf) cavities, or laser modulators in an SSMB storage ring, for longitudinal strong focusing are discussed using the same formalism with important observations made.

\section{COURANT-SNYDER FORMALISM}

The quantum diffusion in $z$ in a storage ring emphasizes the fact that we should consider the quantum excitation on the longitudinal emittance rather than that on the particle 
energy alone. The key to understanding the effect is to change from the global viewpoint to a local one; i.e., the quantum excitation at different places around the ring contributes to the longitudinal emittance with different strengths, just like its transverse counterpart. For an accurate analysis of this effect, here we apply Chao's SLIM formalism [14]. 6D state vector $\mathbf{X}=\left(x, x^{\prime}, y, y^{\prime}, z, \delta\right)^{T}$ of the electron is used, with the superscript $T$ representing the transpose. According to SLIM, the equilibrium eigenemittances and second moments of the beam in an electron storage ring are

$$
\epsilon_{k}=\left\langle\left|A_{k}\right|^{2}\right\rangle=\left\langle\left|A_{-k}\right|^{2}\right\rangle=\frac{C_{L} \gamma^{5}}{c \alpha_{k}} \oint \frac{\left|\mathbf{E}_{k 5}(s)\right|^{2}}{|\rho(s)|^{3}} d s
$$

and

$$
\boldsymbol{\Sigma}_{i j}(s)=\left\langle\mathbf{X}_{i} \mathbf{X}_{j}\right\rangle(s)=2 \sum_{k=I, I I, I I I} \epsilon_{k} \operatorname{Re}\left[\mathbf{E}_{k i}(s) \mathbf{E}_{k j}^{*}(s)\right],
$$

respectively, where $c$ is the speed of light in free space, $\alpha_{k}$ are the damping constants of the three eigenmodes, $C_{L}=55 r_{e} \hbar /\left(48 \sqrt{3} m_{e}\right)$, with $\hbar$ the reduced Planck's constant, $r_{e}$ the electron classical radius, and $m_{e}$ the electron rest mass, $\mathbf{E}_{k}$ are the eigenvectors of the linear one-turn map $\mathbf{M}$, * denotes complex conjugate, and $\operatorname{Re}[]$ means taking the real part of a complex number. The eigenemittances $\epsilon_{k}$ are defined as the positive eigenvalues of $i \boldsymbol{\Sigma} \mathbf{S}$, where $i$ is the imaginary unit, $\boldsymbol{\Sigma}=\left\langle\mathbf{X} \mathbf{X}^{T}\right\rangle$ is the second moments matrix, and $\mathbf{S}$ is the symplectic form [15]. The subscripts $I, I I, I I I$ represent the three eigenmodes and in the planar uncoupled case correspond to the usual horizontal, vertical, and longitudinal dimensions $x, y, z$.

To simplify the discussion, here we consider only the horizontal and longitudinal dimensions and use the state vector $\mathbf{X}=\left(x, x^{\prime}, z, \delta\right)^{T}$. Under the assumptions that the ring is planar uncoupled and the rfs are placed at dispersion-free locations, which is the typical setup for present synchrotron sources, the betatron coordinate $\mathbf{X}_{\beta}=\mathbf{B X}$ can be introduced to parametrize the transfer matrix in a diagonal form, with the dispersion matrix

$$
\mathbf{B}=\left(\begin{array}{cccc}
1 & 0 & 0 & -D_{x} \\
0 & 1 & 0 & -D_{x}^{\prime} \\
D_{x}^{\prime} & -D_{x} & 1 & 0 \\
0 & 0 & 0 & 1
\end{array}\right) .
$$

The one-turn map $\mathbf{M}$ of $\mathbf{X}$ is related to the one-turn map $\mathbf{M}_{\beta}$ of $\mathbf{X}_{\beta}$ by

$$
\mathbf{M}=\mathbf{B}^{-1} \mathbf{M}_{\beta} \mathbf{B}, \quad \mathbf{M}_{\beta}=\left(\begin{array}{cc}
\mathbf{M}_{x \beta} & 0 \\
0 & \mathbf{M}_{z \beta}
\end{array}\right),
$$

with
$\mathbf{M}_{x, z \beta}=\left(\begin{array}{cc}\cos \Phi_{x, z}+\alpha_{x, z} \sin \Phi_{x, z} & \beta_{x, z} \sin \Phi_{x, z} \\ -\gamma_{x, z} \sin \Phi_{x, z} & \cos \Phi_{x, z}-\alpha_{x, z} \sin \Phi_{x, z}\end{array}\right)$,

in which $\Phi_{x}=2 \pi \nu_{x}$ and $\Phi_{z}=2 \pi \nu_{s}$ are the betatron and synchrotron phase advance per turn, respectively. The eigenvectors of $\mathbf{M}_{\beta}$ can be expressed using the CourantSnyder functions [16] as

$$
\mathbf{v}_{x}=\frac{1}{\sqrt{2}}\left(\begin{array}{c}
\sqrt{\beta_{x}} \\
\frac{i-\alpha_{x}}{\sqrt{\beta_{x}}} \\
0 \\
0
\end{array}\right), \quad \mathbf{v}_{z}=\frac{1}{\sqrt{2}}\left(\begin{array}{c}
0 \\
0 \\
\sqrt{\beta_{z}} \\
\frac{i-\alpha_{z}}{\sqrt{\beta_{z}}}
\end{array}\right) \text {. }
$$

Therefore, the eigenvectors of $\mathbf{M}$ are

$$
\begin{aligned}
& \mathbf{E}_{x}=\mathbf{B}^{-1} \mathbf{v}_{x}=\frac{1}{\sqrt{2}}\left(\begin{array}{c}
\sqrt{\beta_{x}} \\
\frac{i-\alpha_{x}}{\sqrt{\beta_{x}}} \\
-\sqrt{\beta_{x}} D_{x}^{\prime}+\frac{i-\alpha_{x}}{\sqrt{\beta_{x}}} D_{x} \\
0
\end{array}\right), \\
& \mathbf{E}_{z}=\mathbf{B}^{-1} \mathbf{v}_{z}=\frac{1}{\sqrt{2}}\left(\begin{array}{c}
\frac{i-\alpha_{z}}{\sqrt{\beta_{z}}} D_{x} \\
\frac{i-\alpha_{z}}{\sqrt{\beta_{z}}} D_{x}^{\prime} \\
\sqrt{\beta_{z}} \\
\frac{i-\alpha_{z}}{\sqrt{\beta_{z}}}
\end{array}\right) .
\end{aligned}
$$

According to SLIM, the equilibrium horizontal and longitudinal emittance are then

$$
\begin{aligned}
\epsilon_{x} & =\frac{55}{96 \sqrt{3}} \frac{\alpha_{F} \chi_{e}^{2} \gamma^{5}}{\alpha_{H}} \oint \frac{\mathcal{H}_{x}(s)}{|\rho(s)|^{3}} d s, \\
\epsilon_{z} & =\frac{55}{96 \sqrt{3}} \frac{\alpha_{F} \chi_{e}^{2} \gamma^{5}}{\alpha_{L}} \oint \frac{\beta_{z}(s)}{|\rho(s)|^{3}} d s,
\end{aligned}
$$

respectively, in which $\alpha_{H}$ and $\alpha_{L}$ are the horizontal and longitudinal damping constants, respectively, $\alpha_{F}=\frac{1}{137}$ is the fine structure constant, $\chi_{e}=\lambda_{e} / 2 \pi=386 \mathrm{fm}$ is the reduced Compton wavelength of the electron, and $\mathcal{H}_{x}=$ $\gamma_{x} D_{x}^{2}+2 \alpha_{x} D_{x} D_{x}^{\prime}+\beta_{x} D_{x}^{\prime 2}$ is the horizontal chromatic function.

After getting the eigenemittances, we can obtain the second moments of the beam according to Eqs. (2) and (7); more specifically, 


$$
\begin{aligned}
\boldsymbol{\Sigma} & =\mathbf{B}^{-1} \boldsymbol{\Sigma}_{\beta}\left(\mathbf{B}^{-1}\right)^{T} \\
& =\left(\begin{array}{cccc}
\epsilon_{x} \beta_{x}+\epsilon_{z} \gamma_{z} D_{x}^{2} & -\epsilon_{x} \alpha_{x}+\epsilon_{z} \gamma_{z} D_{x} D_{x}^{\prime} & -\left(\epsilon_{x} \alpha_{x}+\epsilon_{z} \alpha_{z}\right) D_{x}-\epsilon_{x} \beta_{x} D_{x}^{\prime} & \epsilon_{z} \gamma_{z} D_{x} \\
-\epsilon_{x} \alpha_{x}+\epsilon_{z} \gamma_{z} D_{x} D_{x}^{\prime} & \epsilon_{x} \gamma_{x}+\epsilon_{z} \gamma_{z} D_{x}^{\prime 2} & \epsilon_{x} \gamma_{x} D_{x}+\left(\epsilon_{x} \alpha_{x}-\epsilon_{z} \alpha_{z}\right) D_{x}^{\prime} & \epsilon_{z} \gamma_{z} D_{x}^{\prime} \\
-\left(\epsilon_{x} \alpha_{x}+\epsilon_{z} \alpha_{z}\right) D_{x}-\epsilon_{x} \beta_{x} D_{x}^{\prime} & \epsilon_{x} \gamma_{x} D_{x}+\left(\epsilon_{x} \alpha_{x}-\epsilon_{z} \alpha_{z}\right) D_{x}^{\prime} & \epsilon_{z} \beta_{z}+\epsilon_{x} \mathcal{H}_{x} & -\epsilon_{z} \alpha_{z} \\
\epsilon_{z} \gamma_{z} D_{x} & \epsilon_{z} \gamma_{z} D_{x}^{\prime} & -\epsilon_{z} \alpha_{z} & \epsilon_{z} \gamma_{z}
\end{array}\right),
\end{aligned}
$$

where $\boldsymbol{\Sigma}_{\beta}=\left\langle\mathbf{X}_{\beta} \mathbf{X}_{\beta}^{T}\right\rangle$. The distribution of a Gaussian beam is related to the second moments matrix according to

$$
\begin{aligned}
\psi(\mathbf{X}) & =\frac{1}{(2 \pi)^{2} \sqrt{\operatorname{det} \boldsymbol{\Sigma}}} \exp \left(-\frac{1}{2} \mathbf{X}^{T} \boldsymbol{\Sigma}^{-1} \mathbf{X}\right) \\
& =\frac{1}{(2 \pi)^{2} \epsilon_{x} \epsilon_{z}} \exp \left(-\frac{I_{x}}{\epsilon_{x}}-\frac{I_{z}}{\epsilon_{z}}\right)
\end{aligned}
$$

with $\quad I_{x}=\left[\gamma_{x}\left(x-D_{x} \delta\right)^{2}+2 \alpha_{x}\left(x-D_{x} \delta\right)\left(x^{\prime}-D_{x}^{\prime} \delta\right)+\right.$ $\left.\beta_{x}\left(x^{\prime}-D_{x}^{\prime} \delta\right)^{2}\right] / 2$ and $I_{z}=\left[\gamma_{z}\left(z+D_{x}^{\prime} x-D_{x} x^{\prime}\right)^{2}+\right.$ $\left.2 \alpha_{z}\left(z+D_{x}^{\prime} x-D_{x} x^{\prime}\right) \delta+\beta_{z} \delta^{2}\right] / 2$.

\section{SINGLE rf}

\section{A. Classical $\sqrt{|\eta|}$ scaling}

Now we first reproduce the classical $\sigma_{z} \propto \sqrt{|\eta|}$ scaling using this longitudinal Courant-Snyder parameterization. To simplify the discussion further, in this section and the following, we focus on the longitudinal dimension only and the state vector $\mathbf{X}=(z, \delta)^{T}$ is used. We treat first the case where there is only one rf placed at a dispersion-free location. In this case, the linear longitudinal one-turn map in the middle of the rf cavity is

$$
\begin{aligned}
\mathbf{M} & =\left(\begin{array}{cc}
1 & 0 \\
\frac{h}{2} & 1
\end{array}\right)\left(\begin{array}{cc}
1 & -\eta C_{0} \\
0 & 1
\end{array}\right)\left(\begin{array}{ll}
1 & 0 \\
\frac{h}{2} & 1
\end{array}\right) \\
& =\left(\begin{array}{cc}
1-\frac{h}{2} \eta C_{0} & -\eta C_{0} \\
h-\left(\frac{h}{2}\right)^{2} \eta C_{0} & 1-\frac{h}{2} \eta C_{0}
\end{array}\right)
\end{aligned}
$$

with $h=e V_{\mathrm{rf}} k_{\mathrm{rf}} \cos \phi_{s} / E_{0}$ quantifying the rf gradient, where $e$ is the elementary charge, $V_{\mathrm{rf}}$ is the rf voltage, $k_{\mathrm{rf}}=2 \pi / \lambda_{\mathrm{rf}}$ is the $\mathrm{rf}$ wave number, $\phi_{s}$ is the synchronous phase, and $E_{0}=\gamma m_{e} c^{2}$ is the electron energy. The $R_{56}=-\eta C_{0}$, a measure for the dependence of $z$ on $\delta$, of the ring and the rf kick $h$ can be viewed as the longitudinal drift space and quadrupole, in correspondence to their transverse counterparts, respectively. Note, however, that the $R_{56}$ can be either positive or negative, while the physical length of a drift space is always positive.

For rings working in the longitudinal weak focusing regime, $\left|\nu_{s}\right| \ll 1$, we then have

$$
\begin{aligned}
1-\frac{h}{2} \eta C_{0} & =\cos \Phi_{z} \approx 1-\frac{\Phi_{z}^{2}}{2} \Rightarrow \Phi_{z} \\
& \approx \begin{cases}-\sqrt{h \eta C_{0}} & \text { if } \eta>0, \\
\sqrt{h \eta C_{0}} & \text { if } \eta<0 .\end{cases}
\end{aligned}
$$

Therefore, $\beta_{z}$ at the $\mathrm{rf}$ center is

$$
\beta_{z \mathrm{~S}}=\frac{\mathbf{M}_{12}}{\sin \Phi_{z}} \approx \frac{-\eta C_{0}}{\Phi_{z}} \approx \sqrt{\eta C_{0} / h} .
$$

Here, we use the subscript $\mathrm{S}$ to denote that the result is the same with that obtained in Sands' analysis [1], although the method used here is different. As $\left|\nu_{s}\right| \ll 1$, therefore,

$$
\beta_{z \mathrm{~S}} \gg\left|-\eta C_{0}\right|
$$

We will see later in Sec. IV that, in a longitudinal strong focusing ring, $\left|\nu_{s}\right|$ can be close to or even larger than 1 , and $\beta_{z}$ then can be the same level of or smaller than $\left|-\eta C_{0}\right|$.

Using this $\beta_{z S}$ to represent $\beta_{z}$ of the whole ring, we then get the longitudinal emittance obtained in Sands' analysis [1]:

$$
\epsilon_{z \mathrm{~S}}=\frac{55}{96 \sqrt{3}} \frac{\alpha_{F} \chi_{e}^{2} \gamma^{5} \beta_{z \mathrm{~S}}}{\alpha_{L}} \oint \frac{1}{|\rho(s)|^{3}} d s
$$

For a ring consisting of isomagnets which bend always inward, $\rho$ a positive constant and $\alpha_{L}=J_{z} U_{0} / 2 E_{0}=$ $J_{z} 2 \pi \AA_{e} \alpha_{F} \gamma^{3} / 3 \rho$, with $U_{0}$ the radiation loss per turn and $J_{z}$ the longitudinal damping partition number [1], we have

$$
\begin{aligned}
& \epsilon_{z \mathrm{~S}} \approx \frac{55 \chi_{e}}{J_{z} 32 \sqrt{3}} \frac{\gamma^{2}}{\rho} \sqrt{\frac{\eta C_{0}}{h}} \approx \sigma_{\delta \mathrm{S}}^{2} \beta_{z \mathrm{~S}}, \\
& \sigma_{z \mathrm{~S}}=\sqrt{\epsilon_{z \mathrm{~S}} \beta_{z \mathrm{~S}}} \approx \sqrt{\frac{55 \chi_{e}}{J_{z} 32 \sqrt{3}} \frac{\gamma^{2}}{\rho}} \sqrt{\frac{\eta C_{0}}{h}} \approx \sigma_{\delta \mathrm{S}} \beta_{z \mathrm{~S}}, \\
& \sigma_{\delta \mathrm{S}}=\sqrt{\epsilon_{z \mathrm{~S}} \gamma_{z \mathrm{~S}}} \approx \sqrt{\epsilon_{z \mathrm{~S}} / \beta_{z \mathrm{~S}}} \approx \sqrt{\frac{55 \lambda_{e}}{J_{z} 32 \sqrt{3}} \frac{\gamma^{2}}{\rho}} .
\end{aligned}
$$

Therefore, to generate short bunches in an electron storage ring, we need to implement a quasi-isochronous lattice and high rf gradient. 


\section{B. Beyond the classical $\sqrt{|\eta|}$ scaling}

Using a single $\beta_{z S}$ to represent that of the whole ring is valid in usual rings where the relative variation of $\beta_{z}$ is negligible and the electron distribution in the longitudinal phase space is always upright. But when $\eta$ is small, the partial phase slippage can be significantly larger than the global one, and the variation of $\beta_{z}$ and beam orientation in the longitudinal phase space around the ring can be significant; thus, the classical $\sqrt{|\eta|}$ scaling fails. Now we use the longitudinal Courant-Snyder formalism to conduct an analysis of this. At a position $s_{j}$, the ring can be divided into three parts, with their transfer matrices given by

$$
\begin{aligned}
\mathbf{T}\left(s_{j}, s_{\mathrm{rf}-}\right) & =\left(\begin{array}{cc}
1 & F\left(s_{j}, s_{\mathrm{rf}-}\right) \\
0 & 1
\end{array}\right), \\
\mathbf{T}\left(s_{\mathrm{rf}-}, s_{\mathrm{rf}+}\right) & =\left(\begin{array}{ll}
1 & 0 \\
h & 1
\end{array}\right), \\
\mathbf{T}\left(s_{\mathrm{rf}+}, s_{j}\right) & =\left(\begin{array}{cc}
1 & F\left(s_{\mathrm{rf}+}, s_{j}\right) \\
0 & 1
\end{array}\right),
\end{aligned}
$$

where

$$
F\left(s_{1}, s_{2}\right)=-\int_{s_{1}}^{s_{2}}\left(\frac{D_{x}(s)}{\rho(s)}-\frac{1}{\gamma^{2}}\right) d s
$$

is the partial $R_{56}$ from $s_{1}$ to $s_{2}$ and

$$
F\left(s_{j}, s_{\mathrm{rf}-}\right)+F\left(s_{\mathrm{rf}+}, s_{j}\right)=-\eta C_{0},
$$

with $+(-)$ denoting right after (before) the corresponding location. The one-turn map at $s_{j}$ is

$$
\mathbf{M}\left(s_{j}\right)=\mathbf{T}\left(s_{\mathrm{rf}+}, s_{j}\right) \mathbf{T}\left(s_{\mathrm{rf}-}, s_{\mathrm{rf}+}\right) \mathbf{T}\left(s_{j}, s_{\mathrm{rf}-}\right) .
$$

Therefore,

$$
\begin{aligned}
\beta_{z}\left(s_{j}\right) & =\frac{\mathbf{M}_{12}\left(s_{j}\right)}{\sin \Phi_{z}} \\
& =\frac{-\eta C_{0}+F\left(s_{\mathrm{rf}+}, s_{j}\right) F\left(s_{j}, s_{\mathrm{rf}-}\right) h}{\sin \Phi_{z}} .
\end{aligned}
$$

As can be seen, the first term in the numerator is the usual global phase slippage $\left(R_{56}\right)$ of the ring. The second term represents the impact of variation of the partial phase slippage on $\beta_{z}$. Sands' analysis means to drop the second term but keep only the first term. With both terms considered, the more accurate longitudinal emittance is then

$$
\begin{array}{r}
\epsilon_{z}=\epsilon_{z \mathrm{~S}}\left(1+\frac{\left\langle F^{2}\left(s_{\mathrm{rf}+}, s_{j}\right)\right\rangle+\eta C_{0}\left\langle F\left(s_{\mathrm{rf}+}, s_{j}\right)\right\rangle}{\eta C_{0} / h}\right) \\
\approx \epsilon_{z \mathrm{~S}}\left(1+\frac{\left\langle F^{2}\left(s_{\mathrm{rf}+}, s_{j}\right)\right\rangle+\eta C_{0}\left\langle F\left(s_{\mathrm{rf}+}, s_{j}\right)\right\rangle}{\beta_{z \mathrm{~S}}^{2}}\right),
\end{array}
$$

in which $\langle P\rangle$ means the radiation-weighted average of a parameter $P$ around the ring, defined as

$$
\langle P\rangle=\frac{\oint \frac{P}{|\rho(s)|^{3}} d s}{\oint \frac{1}{|\rho(s)|^{3}} d s} ;
$$

i.e., the average actually takes place only at the dipoles. After getting the longitudinal emittance and Courant-Snyder functions, the bunch length and energy spread at $s_{j}$ can be obtained by

$$
\sigma_{z}\left(s_{j}\right)=\sqrt{\epsilon_{z} \beta_{z}\left(s_{j}\right)}, \quad \sigma_{\delta}\left(s_{j}\right)=\sqrt{\epsilon_{z} \gamma_{z}\left(s_{j}\right)} .
$$

For the bunch length, more accurately,

$$
\sigma_{z}\left(s_{j}\right)=\sqrt{\epsilon_{z} \beta_{z}\left(s_{j}\right)+\epsilon_{x} \mathcal{H}_{x}\left(s_{j}\right)},
$$

considering the coupling from the transverse emittance [17] according to Eq. (9). In a planar uncouple ring which means there is only passive transverse-longitudinal coupling introduced by bending magnets, the transverse emittance always lengthens the bunch at places where $\mathcal{H}_{x} \neq 0$. But this conclusion is not generally true if we take advantage of transverse-longitudinal coupling actively for bunch compression when the transverse emittance is small [18-20]. The reason is that there is flexibility in projecting the three eigenemittances of a beam into different physical dimensions as shown in Eq. (2), although their values cannot be changed in a linear symplectic lattice [21].

As analyzed before, we know that in a longitudinal weak focusing ring $\epsilon_{z \mathrm{~S}} \propto \sqrt{\eta / h}$, but now, according to Eq. (22), there exists a specific $|\eta|$ where a minimum $\epsilon_{z}$ will be reached when adjusting $|\eta|$ by changing the dispersion function slightly if the overall pattern is given. Below this $|\eta|, \epsilon_{z}$ will grow with the decrease of $|\eta|$. The same argument applies also to the rf gradient $h$. Above a specific $|h|$, the emittance will grow, instead of decreasing, with the increase of $|h|$.

The above analysis has been confirmed by particle tracking simulation using a low-alpha lattice of the Metrology Light Source (MLS) storage ring [22,23] with the dispersion function pattern shown in Fig. 1. Also shown are $\beta_{z}(s)$ (for the case of $V_{\mathrm{rf}}=80 \mathrm{MV}$ ) and $F(0, s)$ around the ring. The other related parameters for the simulation are given in Table I. The beam energy used is higher than the actual operation value of the MLS, and the rf voltages applied is also dramatically high. They are chosen here for 


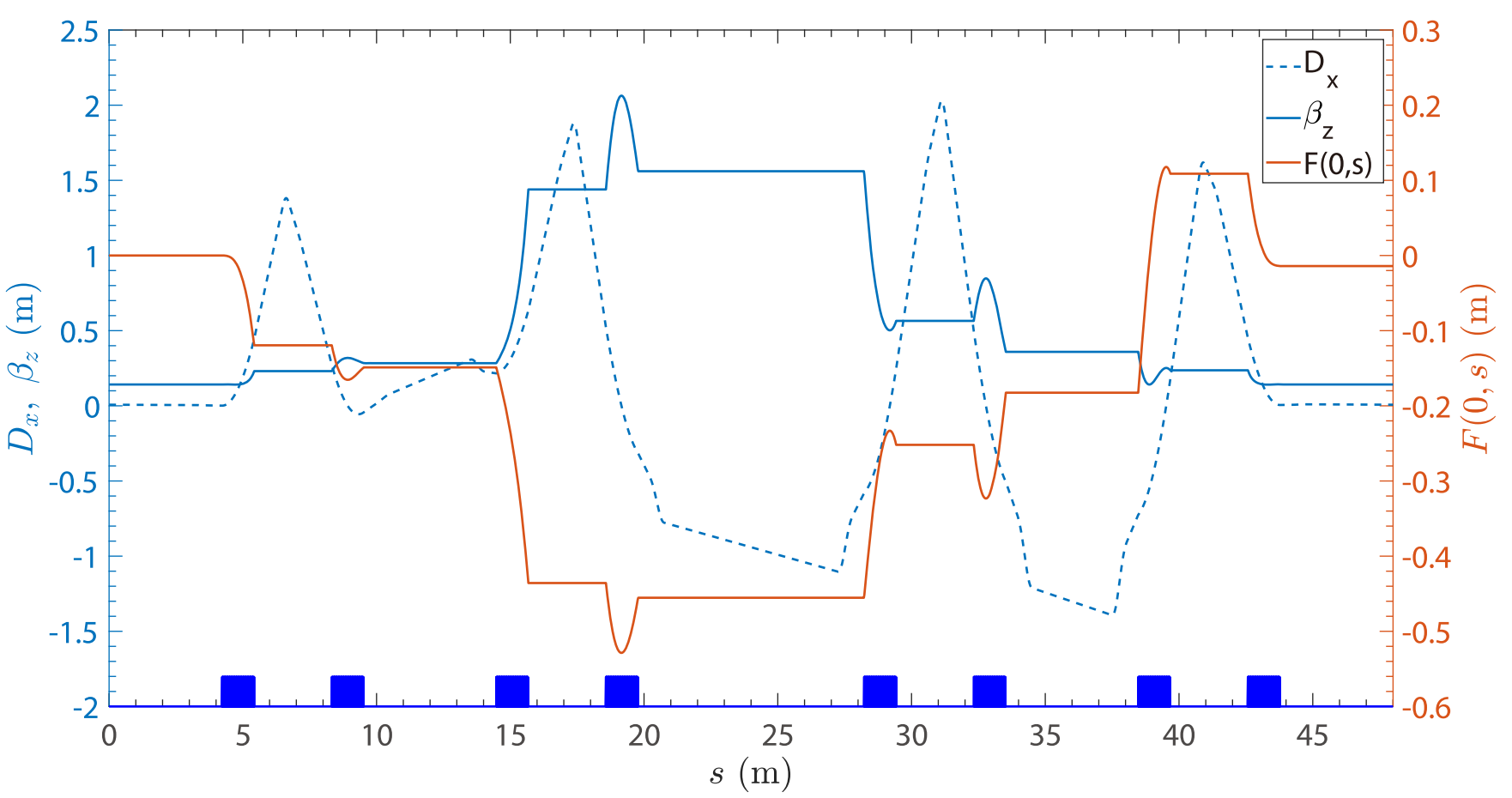

FIG. 1. The horizontal dispersion $D_{x}$, longitudinal beta function $\beta_{z}$, and $F(0, s)$ of the MLS lattice used for analysis and simulation. In this plot, the zero-length $\mathrm{rf}$ is placed at $s_{\mathrm{rf}}=0 \mathrm{~m}$ and $V_{\mathrm{rf}}=80 \mathrm{MV}$ is applied. The dipoles are shown at the bottom as blue rectangles. Each dipole has a length of $1.2 \mathrm{~m}$ and bends the electron trajectory for an angle of $\pi / 4$.

the numerical example to present the difference of this work and the classical analysis of Sands in a more notable way. The simulation results along with the theoretical predictions from Sands' analysis and this paper are shown in Fig. 2. Also shown is the theoretical calculation based on SLIM [14]. As can be seen from Fig. 2, our result and SLIM agree well with the simulation in the cases of ultrahigh $\mathrm{rf}$ voltages, while the predication of Sands deviates from the simulation.

TABLE I. Parameters of the MLS lattice used in simulation.

\begin{tabular}{lll}
\hline \hline Parameter & \multicolumn{1}{c}{ Value } & \multicolumn{1}{c}{ Description } \\
\hline$E_{0}$ & $1.2 \mathrm{GeV}$ & Beam energy \\
$C_{0}$ & $48 \mathrm{~m}$ & Ring circumference \\
$\eta$ & $2.9 \times 10^{-4}$ & Phase slippage factor \\
$f_{\mathrm{rf}}$ & $500 \mathrm{MHz}$ & rf frequency \\
$V_{\mathrm{rf}}$ & $2.5-160 \mathrm{MV}$ & rf voltage \\
$U_{0}$ & $0.12 \mathrm{MeV}$ & Radiation loss per turn \\
$J_{z}$ & 1.94 & Longitudinal damping partition \\
$\tau_{\delta}$ & $1.6 \mathrm{~ms}$ & Radiation damping time \\
$\sigma_{\delta \mathrm{S}}$ & $8.4 \times 10^{-4}$ & Natural energy spread \\
$\left\langle F\left(s_{\mathrm{rf}}, s_{j}\right)\right\rangle$ & $-0.19 \mathrm{~m}$ & $\cdots$ \\
$\sqrt{\left\langle F^{2}\left(s_{\mathrm{rf}}, s_{j}\right)\right\rangle}$ & $0.26 \mathrm{~m}$ & $\cdots$ \\
$\sqrt{\left\langle F^{2}\right\rangle-\langle F\rangle^{2}}$ & $0.18 \mathrm{~m}$ & $\ldots$ \\
\hline \hline
\end{tabular}

\section{Discussions}

Now we make some more discussions. First, we observe that in Fig. $1 \beta_{z}$ changes notably only inside dipoles, which reflects the fact that $F(0, s)$, i.e., the effective longitudinal drift space, changes significantly only inside dipoles where $\rho \neq 0$. The minimum $\beta_{z}$ around the ring is realized when $F\left(s_{\text {rf }+}, s_{j}\right)=F\left(s_{j}, s_{\text {rf }-}\right)=\frac{-\eta C_{0}}{2}$, and

$$
\beta_{z, \min }=\left(1-\frac{h \eta C_{0}}{4}\right) \beta_{z \mathrm{~S}}
$$

Furthermore, in some cases, $\epsilon_{z}$ can actually be smaller than $\epsilon_{z \mathrm{~S}}$. The physical meaning is then the average $\beta_{z}$ at the dipoles is smaller than that at the rf. This, however, is usually not satisfied in a low-alpha lattice, where the change of $F\left(s_{\mathrm{rf}+}, s_{j}\right)$ around the ring can be significantly larger than the global ring $R_{56}=-\eta C_{0}$. Therefore, $\beta_{z}$ at other places can be significantly larger than that at the rf, just like the case shown in Fig. 1. For a novel light source mechanism like SSMB [4-13], an ultrasmall $\epsilon_{z}$ is desired. According to Eq. (8), a method to tailor the $\beta_{z}$ around the ring can be envisioned for such a purpose [13], similar to the tailoring of $\mathcal{H}_{x}$ for the minimization of horizontal emittance. The theoretical minimum emittance (TME) can be analyzed, and the same scaling of $\epsilon_{z, \mathrm{TME}} \propto \gamma^{2} \theta^{3}$, where $\theta$ is the bending angle of each dipole, can be derived similar to the case of transverse dimension. Generally, a small 


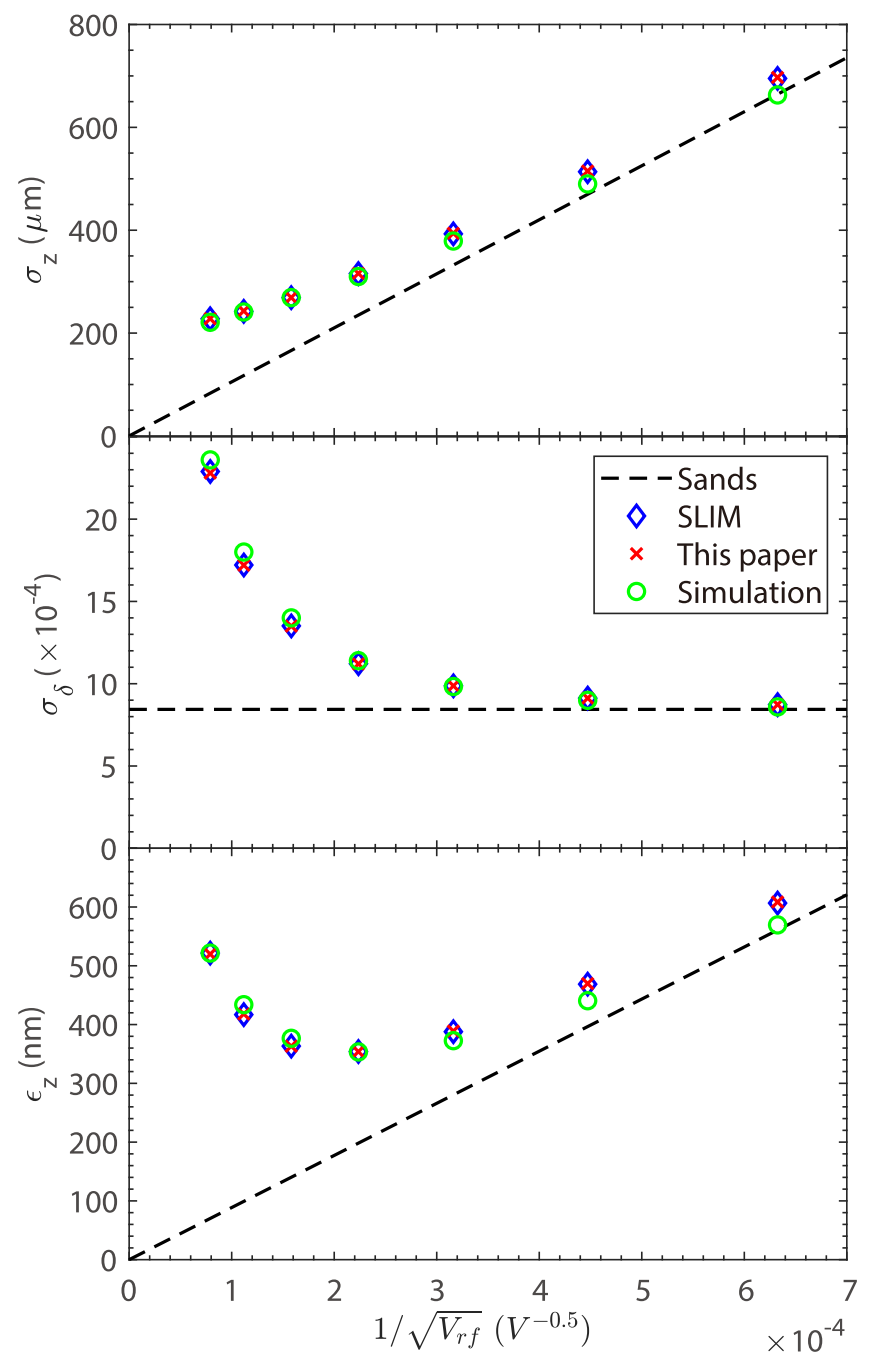

FIG. 2. Bunch length saturation and energy spread and longitudinal emittance divergence when the bunch length is pushed to the limit given by quantum diffusion, in the simulation by increasing the rf voltage. The lattice optics and related parameters are shown in Fig. 1 and Table I. In each simulation, 1000 electrons are tracked for 50000 turns, which corresponds to about five longitudinal damping times, and the averaged result of the last 10000 turns are used to get the $\sigma_{z}, \sigma_{\delta}$, and $\epsilon_{z}$. The observation point is at the rf.

longitudinal emittance requires a small dipole bending angle and small dispersion at the dipoles. The application of transverse and longitudinal gradient bends, etc., can also be anticipated for reducing $\epsilon_{z}$.

We point out that quantifying the impact of variation of $\beta_{z}$ around the ring on $\epsilon_{z}$ using partial alpha or partial phase slippage variance $\left\langle F^{2}\right\rangle-\langle F\rangle^{2}$, as that done in Ref. [2] and also our previous publication [11], is not generally accurate. Given the same dispersion function pattern, which means the same $\left\langle F^{2}\right\rangle-\langle F\rangle^{2}$ as it is independent of the observation point, a different longitudinal beta function pattern can be generated if the rf is placed at a different location, therefore resulting in a different longitudinal emittance according to Eq. (8).
We can also view this from another way. Changing the rf location means shifting $F\left(s_{\mathrm{rf}+}, s_{j}\right)$ up or down as a whole, which can also be done by artificially inserting a pair of $\pm r_{56}$ besides the rf. We assume that there is no synchrotron radiation within these artificially inserted $\pm r_{56}$, and, therefore, these manipulations do not change the $R_{56}$ and also $\left\langle F^{2}\right\rangle-\langle F\rangle^{2}$ of the ring, but they do affect $\beta_{z}$ and, therefore, also $\epsilon_{z}$. When $r_{56}=\left\langle F\left(s_{\mathrm{rf}+}, s_{j}\right)\right\rangle+\frac{\eta C_{0}}{2}$, we arrive at the minimum longitudinal emittance

$$
\epsilon_{z, \min }=\epsilon_{z \mathrm{~S}}\left(1+\frac{\left\langle F^{2}\right\rangle-\langle F\rangle^{2}-\left(\frac{\eta C_{0}}{2}\right)^{2}}{\eta C_{0} / h}\right)
$$

Equivalently, in the case of shifting rf location, $\epsilon_{z, \min }$ is reached when the $\mathrm{rf}$ is placed at a location such that $\left\langle F\left(s_{\mathrm{rf}+}, s_{j}\right)\right\rangle=-\frac{\eta C_{0}}{2}$. The maximum longitudinal emittance is realized when the $\mathrm{rf}$ is placed at a place such that $\left|\left\langle F\left(s_{\mathrm{rf}+}, s_{j}\right)\right\rangle+\frac{\eta C_{0}}{2}\right|$ reaches the maximum possible value.

To give the readers a more concrete feeling, here we present some calculations based on the lattice optics presented in Fig. 1 and other related parameters given in Table I. Figure 3 shows the emittance ratio $\epsilon_{z} / \epsilon_{z \mathrm{~S}}$ as a
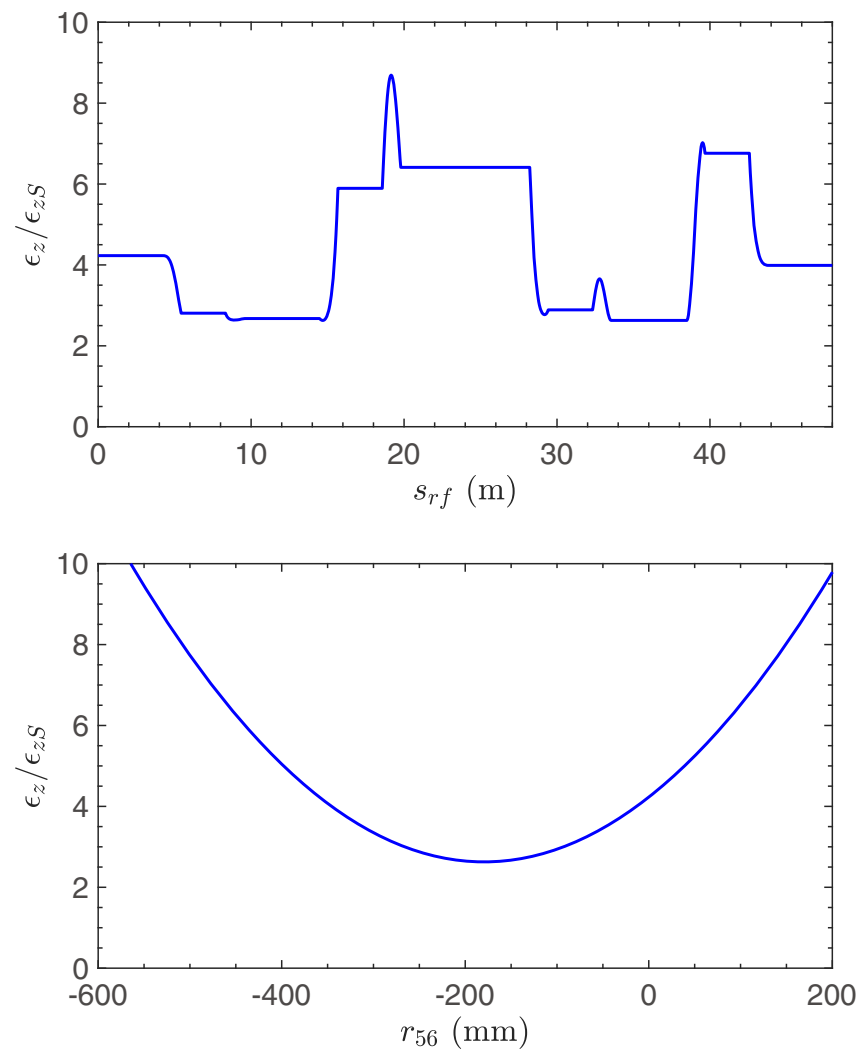

FIG. 3. The emittance ratio $\frac{\epsilon_{z}}{\epsilon_{\mathrm{S}}}$ as a function of the rf place $s_{\text {rf }}$ (top) and the $r_{56}$ artificially inserted in front of the rf whose location is fixed at $s_{\mathrm{rf}}=0 \mathrm{~m}$ (bottom). The lattice optics and related parameters are shown in Fig. 1 and Table I. The rf voltage applied is $V_{\mathrm{rf}}=80 \mathrm{MV}$. 
function of the rf place $s_{\mathrm{rf}}$ and the $\pm r_{56}$ artificially inserted besides the rf whose location is fixed at $s_{\mathrm{rf}}=0 \mathrm{~m}$, respectively. An $80 \mathrm{MV}$ rf voltage is applied in both calculations. As can be seen, indeed the place of the rf can have a crucial impact on the longitudinal emittance in this specific lattice optics and choice of rf voltage, and the impact of the artificially inserted $\pm r_{56}$ is also as expected.

When the minimum longitudinal emittance is reached, the bunch length at the rf is

$$
\sigma_{z, \min }\left(s_{\mathrm{rf}}\right)=\sigma_{\delta \mathrm{S}} \sqrt{\frac{\eta C_{0}}{h}+\left\langle F^{2}\right\rangle-\langle F\rangle^{2}-\left(\frac{\eta C_{0}}{2}\right)^{2}} .
$$

In the case of ultrasmall $\eta$,

$$
\sigma_{z, \min }\left(s_{\mathrm{rf}}\right) \approx \sigma_{\delta \mathrm{S}} \sqrt{\left\langle F^{2}\right\rangle-\langle F\rangle^{2}}
$$

Therefore, the variance of $F$ can be viewed as a parameter to quantify the lowest possible contribution of this effect to the equilibrium bunch length at the rf with a dispersion function pattern given [2], if we can choose the location of the rf. However, in a real machine, the rf location is fixed, and Eqs. (22), (24), and (25) should be used instead. The analysis of shifting $\mathrm{rf}$ location or adding artificial $\pm r_{56}$ helps us to better understand the physics, and we recognize the fact that the rf can actually not be placed inside dipoles and also that the Courant-Snyder functions lose their welldefined meaning if the rf is placed at a dispersive location.

\section{Campbell's theorem}

Now we explain in more detail why $\left\langle F^{2}\right\rangle-\langle F\rangle^{2}$ is not a generally accurate criterion. The reason is that, while the photon emission process is stochastic, the evolution of $F$ around the ring is deterministic. So the diffusion of $z$ each turn due to quantum excitation is

$$
\left\langle z^{2}\right\rangle-\langle z\rangle^{2}=\left\langle F^{2}\right\rangle\langle\mathcal{N}\rangle\left\langle\frac{u^{2}}{E_{0}^{2}}\right\rangle
$$

instead of

$$
\left\langle z^{2}\right\rangle-\langle z\rangle^{2}=\left(\left\langle F^{2}\right\rangle-\langle F\rangle^{2}\right)\langle\mathcal{N}\rangle\left\langle\frac{u^{2}}{E_{0}^{2}}\right\rangle,
$$

where $F$ is calculated with the final observation location as the ending point, $\langle\mathcal{N}\rangle$ is the expected number of emitted photons, $u$ is the photon energy, and $\left\langle u^{2}\right\rangle$ and later also $\langle u\rangle$ mean the average is taken with respect to the photon energy spectrum. This result can be understood with the help of Campbell's theorem [24].

From this theorem, some expectation result for the Poisson point process follows. For example, for the application in synchrotron radiation, we have $\delta=-\sum_{i} \frac{u_{i}}{E_{0}}$, where $i$ means the $i$ th photon emission. Then, according to Campbell's theorem, we have

$$
\begin{array}{r}
\langle\delta\rangle=-\langle\mathcal{N}\rangle\left\langle\frac{u}{E_{0}}\right\rangle=-T_{\text {dipole }} \dot{\mathcal{N}}\left\langle\frac{u}{E_{0}}\right\rangle, \\
\left\langle\delta^{2}\right\rangle-\langle\delta\rangle^{2}=\langle\mathcal{N}\rangle\left\langle\frac{u^{2}}{E_{0}^{2}}\right\rangle=T_{\text {dipole }} \dot{\mathcal{N}}\left\langle\frac{u^{2}}{E_{0}^{2}}\right\rangle,
\end{array}
$$

where $\dot{\mathcal{N}}$ is the number of photons emitted per unit time in the dipoles and $T_{\text {dipole }}$ is the total time within dipoles. Equation (32) is why $\dot{\mathcal{N}}\left\langle u^{2}\right\rangle$ appears so often in the calculation of energy spread, emittance, etc., in electron storage ring physics. Note that the relation in Eq. (32) holds as long as the radiation is a Poisson point process. It is independent of whether $\langle u\rangle=0$ or not, and is also independent of the detailed spectrum of the photon energy. In other words, the key of a Poisson point process is the randomness in whether there is a kick or not, i.e., the kick number, and not in the randomness of the size of the kicks. The importance of this theorem for electron dynamics was first pointed out by Sands [25]. A proof can be found in the article of Rice [26] and a less rigorous but simpler one in the lecture note of Jowett [27].

Now we can understand Eq. (30) like this. Suppose the rf is the observation point. We divide the ring into many sections, and in each section $F\left(s_{j}, s_{\mathrm{rf}}\right)$ does not change much. Then the change of electron longitudinal coordinate in one turn is $z=\sum_{j} z_{j}$, with $z_{j}=-\sum_{i} F\left(s_{j i}, s_{\mathrm{rf}}\right) \frac{u_{j i}}{E_{0}}$ the contribution due to photon emissions within the section $j$. So, according to Campbell's theorem, the variance of $z_{j}$ is

$$
\operatorname{Var}\left(z_{j}\right)=F^{2}\left(s_{j}, s_{\mathrm{rf}}\right) t_{j} \dot{\mathcal{N}}\left\langle\frac{u^{2}}{E_{0}^{2}}\right\rangle
$$

where $t_{j}$ is the time within the dipoles in section $j$. As the photon emissions in different sections are uncorrelated, then the variance of $z$ is the sum of variance of $z_{j}$ :

$$
\begin{aligned}
\left\langle z^{2}\right\rangle-\langle z\rangle^{2} & =\frac{\sum_{j}\left[F^{2}\left(s_{j}, s_{\mathrm{rf}}\right) t_{j}\right]}{T_{\text {total }}} T_{\text {total }} \dot{\mathcal{N}}\left\langle\frac{u^{2}}{E_{0}^{2}}\right\rangle \\
& =\left\langle F^{2}\left(s_{j}, s_{\mathrm{rf}}\right)\right\rangle\langle\mathcal{N}\rangle\left\langle\frac{u^{2}}{E_{0}^{2}}\right\rangle,
\end{aligned}
$$

in which $T_{\text {total }}=\sum_{j} t_{j}$ is the total time within the dipoles. In fact, the same argument can be applied to explain why $\left|E_{k 5}(s)\right|^{2}$ in Eq. (1), instead of their variance, are used in SLIM to calculate the increase of eigenemittances due to quantum excitation. 


\section{MULTIPLE rfs}

\section{A. Analysis}

The analysis in the above section considers the case with only a single rf. When there are multiple rfs, for the longitudinal dynamics, it is similar to implement multiple quadrupoles in the transverse dimension, and the beam dynamics can have more possibilities. A longitudinal strong focusing scheme, for example, can be invoked $[6,28]$ not unlike its transverse counterpart which almost all the storage rings today implement. The linear beam dynamics with multiple rfs can be treated the same way as that with a single rf using SLIM. When all the rfs are placed at dispersion-free locations, the Courant-Snyder parametrization can be applied as analyzed in Sec. II. Here, we use a setup with two rfs as an example to show the scheme of manipulating $\beta_{z}$ around the ring. The schematic layout of the ring is shown in Fig. 4. The treatment of cases with more rfs is similar.

We divide the ring into five sections, i.e., three longitudinal drifts $\left(R_{56}\right)$ and two longitudinal quadrupole kicks $(h)$, with the transfer matrices given by

$$
\begin{array}{rlr}
\mathbf{T}_{\mathrm{D} 1}=\left(\begin{array}{cc}
1 & R_{56}^{(1)} \\
0 & 1
\end{array}\right), & \mathbf{T}_{\mathrm{rf} 1}=\left(\begin{array}{cc}
1 & 0 \\
h_{1} & 1
\end{array}\right), \\
\mathbf{T}_{\mathrm{D} 2}=\left(\begin{array}{cc}
1 & R_{56}^{(2)} \\
0 & 1
\end{array}\right), & \mathbf{T}_{\mathrm{r} 22}=\left(\begin{array}{cc}
1 & 0 \\
h_{2} & 1
\end{array}\right), \\
\mathbf{T}_{\mathrm{D} 3}=\left(\begin{array}{cc}
1 & R_{56}^{(3)} \\
0 & 1
\end{array}\right) . &
\end{array}
$$

Then the one-turn map at the radiator center is

$$
\mathbf{M}_{\mathrm{R}}=\mathbf{T}_{\mathrm{D} 3} \mathbf{T}_{\mathrm{rf} 2} \mathbf{T}_{\mathrm{D} 2} \mathbf{T}_{\mathrm{rf} 1} \mathbf{T}_{\mathrm{D} 1}
$$

Linear stability requires that the absolute value of $\operatorname{Tr} \mathbf{M}_{R}<2$, where $\operatorname{Tr}$ means the trace of. For generation

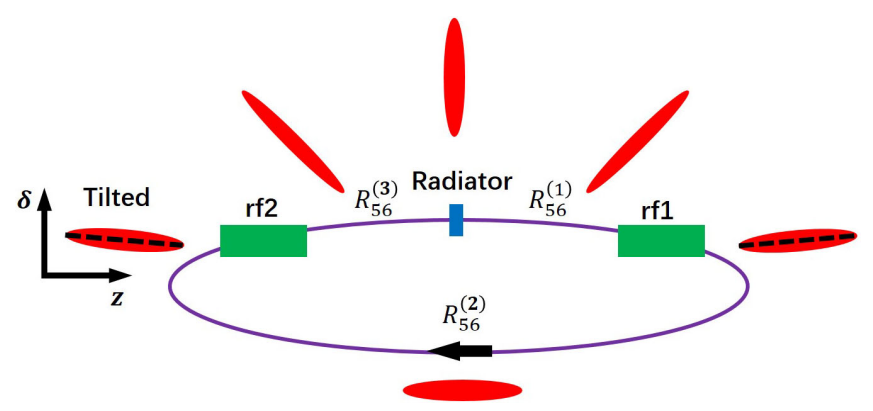

FIG. 4. Schematic layout of a storage ring using two rf systems for longitudinal strong focusing and an example beam distribution evolution in the longitudinal phase space. Note that the tilted angles of the beam distribution and bunch length ratios at different places do not strictly correspond to the parameters in Table II but only to present the qualitative characteristics. of coherent radiation, we usually want the bunch length to reach its minimum at the radiator; then we need $\alpha_{z}=0$ for $\mathbf{M}_{\mathrm{R}}$.

With the primary purpose to present the principle, instead of a detailed design, here for simplicity we discuss only one special case: $R_{56}^{(1)}=R_{56}^{(3)}, h_{1}=h_{2}=h$. The treatment of more general cases with different signs and magnitudes of $R_{56}^{(1)}$ and $R_{56}^{(3)}$ and $h_{1}$ and $h_{2}$ is similar, but the same-signed $R_{56}^{(1)}$ and $R_{56}^{(3)}$ might be easier for a real lattice to fulfill. For example, if $R_{56}^{(1)}, R_{56}^{(3)}>0$, a possible realization of them are chicanes.

For the special case of $R_{56}^{(1)}=R_{56}^{(3)}, h_{1}=h_{2}=h$ and denote $1+R_{56}^{(1)} h=\xi_{1}, 2+R_{56}^{(2)} h=\xi_{2}$, we then have

$$
\mathbf{M}_{\mathrm{R}}=\left(\begin{array}{cc}
\xi_{1} \xi_{2}-1 & \frac{\xi_{1}^{2} \xi_{2}-2 \xi_{1}}{h} \\
h \xi_{2} & \xi_{1} \xi_{2}-1
\end{array}\right) .
$$

The linear stability requires $\left|\xi_{1} \xi_{2}-1\right|<1$, and the synchrotron tune is

$$
\nu_{s}= \begin{cases}\frac{1}{2 \pi} \arccos \left[\xi_{1} \xi_{2}-1\right] & \text { if } \frac{\xi_{1}^{2} \xi_{2}-2 \xi_{1}}{h}>0, \\ 1-\frac{1}{2 \pi} \arccos \left[\xi_{1} \xi_{2}-1\right] & \text { if } \frac{\xi_{1}^{2} \xi_{2}-2 \xi_{1}}{h}<0 .\end{cases}
$$

Here, we give one example parameter set with a stable linear motion as shown in Table II. We recognize the fact that the large energy chirp strength $h$ used is demanding, for SSMB only pulsed laser can now reach such a value, and here our primary goal is to present the principle based on which the interested readers can choose and optimize the parameters for their target applications. According to the longitudinal Courant-Snyder functions given in Table II (note the values of $\beta_{z}$ and the signs of $\alpha_{z}$ ), the evolution of

TABLE II. An example parameter set corresponding to the setup shown in Fig. 4.

\begin{tabular}{ll}
\hline \hline Parameter & \multicolumn{1}{c}{ Value } \\
\hline$R_{56}^{(1)}$ & $15 \mu \mathrm{m}$ \\
$R_{56}^{(2)}$ & $-100 \mu \mathrm{m}$ \\
$R_{56}^{(3)}$ & $15 \mu \mathrm{m}$ \\
$h$ & $-5 \times 10^{4} \mathrm{~m}^{-1}$ \\
$\xi_{1}$ & 0.25 \\
$\xi_{2}$ & 7 \\
$\nu_{s}$ & 0.115 \\
$\beta_{z}\left(s_{\mathrm{rad}}\right)$ & $1.9 \mu \mathrm{m}$ \\
$\beta_{z}\left(s_{\mathrm{rf}}\right)$ & $121 \mu \mathrm{m}$ \\
$\alpha_{z}\left(s_{\mathrm{rad}}\right)$ & 0 \\
$\alpha_{z}\left(s_{\mathrm{rf1-}}\right)$ & -7.9 \\
$\alpha_{z}\left(s_{\mathrm{rf} 1+}\right)$ & -1.9 \\
$\alpha_{z}\left(s_{\mathrm{rf} 2-}\right)$ & 1.9 \\
$\alpha_{z}\left(s_{\mathrm{rf} 2+}\right)$ & 7.9 \\
\hline \hline
\end{tabular}


electron distribution in the longitudinal phase space (note the bunch lengths and orientations) around the ring is qualitatively shown in Fig. 4.

\section{B. Discussions}

Here, we make several observations from the above analysis and numerical example, which we believe are important. First, $\beta_{z}$ in a longitudinal strong focusing ring is at the same level of or even smaller than the ring $\left|R_{56}=-\eta C_{0}\right|$, while in a longitudinal weak focusing ring $\beta_{z} \gg\left|-\eta C_{0}\right|$. Therefore, according to Eq. (8), the equilibrium longitudinal emittance in a longitudinal strong focusing ring can be much smaller than that in a longitudinal weak focusing ring [13]. Together with a smaller $\beta_{z}$, the bunch length can, thus, be much smaller than that in a longitudinal weak focusing ring. This is the reason behind the application of longitudinal strong focusing in SSMB to realize extreme short bunches $[6,13]$.

Second, $\beta_{z}$ changes significantly around the ring in the longitudinal strong focusing regime. Therefore, the bunch length and beam orientation in the longitudinal phase space vary greatly around the ring, as shown qualitatively in Fig. 4. This means the adiabatic approximation cannot be applied for the longitudinal dimension anymore [15]. Actually, the adiabatic approximation also breaks down in the case corresponding to Fig. 1, where the change of $\beta_{z}$ around the ring is significant although the total synchrotron phase advance per turn is small. Therefore, the global tune is not a general criterion in the classification of a ring to be weak focusing or strong focusing. The evolution of $\beta_{z}$ is more relevant.

The breakdown of adiabatic approximation can have crucial impacts on the study of both the single-particle and collective effects. For linear single-particle dynamics, the longitudinal and transverse dimensions should be treated the same way on equal footing, and SLIM formalism can be invoked. The treatment of nonlinear single-particle dynamics is more subtle, as the longitudinal dynamics now is strongly chaotic. For the collective effects, many classical treatments should be reevaluated, and some new formalism needs to be developed. For example, the Haissinski equation [29] for calculating the equilibrium beam distortion cannot be applied directly then. Also, to our knowledge, there is no discussion on coherent synchrotron radiation (CSR)-induced microwave instability in a longitudinal strong focusing ring. The scaling law obtained in the longitudinal weak focusing [30] cannot be applied directly. 3D CSR effects and also the impact of bunch lengthening from the transverse emittance on CSR need more in-depth study. This is especially true for an SSMB ring, considering the fact that the beam width there is much larger than the microbunch length, while the contrary is true in a conventional ring. The contribution from horizontal emittance can easily dominate the bunch length at many places in an SSMB ring. This, on the other hand, will be helpful to suppress unwanted CSR and may also be helpful in mitigating intrabeam scattering (IBS), as extreme short bunches occur only at limited locations. The IBS in a longitudinal strong focusing ring and a general coupled lattice also deserves special attention. To our knowledge, the IBS formalism of Nash [31] and that of Kubo and Oide [32] can be applied for such purposes, as they are based on $6 \times 6$ general transport matrices, while the classical formalism of Piwinski [33] and Bjorken and Mtingwa [34] both adopt approximations which hold only in longitudinal weak focusing rings. An IBS formalism can be developed based on Chao's SLIM formalism [14], in which eigenanalysis has been invoked and applies to 3D general coupled lattice with longitudinal strong focusing.

\section{SUMMARY}

In this paper, we have presented the longitudinal Courant-Snyder formalism for electron storage ring linear dynamics analysis. We used the formalism first to reproduce the classical $\sigma_{z} \propto \sqrt{|\eta|}$ scaling. We then analyzed the case beyond this classical scaling, considering the fact that the longitudinal beta function can change significantly around the ring in a quasi-isochronous ring, due to the variation of partial phase slippage. Formulas for the equilibrium longitudinal emittance, energy spread, and position-dependent bunch length are derived, based on which several important points concerning the analysis are discussed. Finally, we used the same formalism to analyze the application of multiple rfs in which the scheme of longitudinal strong focusing is invoked and make important observations on this new working regime of a storage ring. We expect that the method and discussions presented in this paper will be useful for the storage ring physics, especially the longitudinal dynamics study, and the development of new light sources like steady-state microbunching.

\section{ACKNOWLEDGMENTS}

We thank Yao Zhang and Zhilong Pan for helpful discussions. This work is supported by National Natural Science Foundation of China (NSFC Grant No. 12035010) and Tsinghua University Initiative Scientific Research Program (Grant No. 20191081195).

[1] M. Sands, SLAC Technical Report No. SLAC-121, 1970.

[2] Y. Shoji, H. Tanaka, M. Takao, and K. Soutome, Phys. Rev. E 54, R4556 (1996).

[3] K. Soutome, M. Takao, H. Tanaka, and Y. Shoji, in Proceedings of the 6th European Particle Accelerator Conference (EPAC'98), Stockholm, 1998 (Institute of Physics, London, 1998), pp. 1008-1010.

[4] D. F. Ratner and A. W. Chao, Phys. Rev. Lett. 105, 154801 (2010). 
[5] Y. Jiao, D. F. Ratner, and A. W. Chao, Phys. Rev. Accel. Beams 14, 110702 (2011).

[6] A. Chao, E. Granados, X. Huang, D. Ratner, and H.-W. Luo, in Proceedings of the 7th International Particle Accelerator Conference (IPAC'16), Busan, Korea, 2016 (JACoW, Geneva, 2016), pp. 1048-1053.

[7] C. Tang, X. Deng, A. Chao, W. Huang, T. Rui, J. Feikes, J. Li, M. Ries, A. Hoehl, D. Ratner et al., in Proceedings of the 60th ICFA Advanced Beam Dynamics Workshop on Future Light Sources (FLS'18), Shanghai, China, 2018 (JACoW, Geneva, 2018), pp. 166-170.

[8] Z. Pan, T. Rui, W. Wan, A. Chao, X. Deng, Y. Zhang, W. Huang, and C. Tang, in Proceedings of the 39th International Free Electron Laser Conference (FEL'19), Hamburg, Germany, 2019 (JACoW, Geneva, 2019), pp. 700-703.

[9] C. Li, C. Feng, B. Jiang, and A. Chao, in Proceedings of the 10th International Particle Accelerator Conference (IPAC'19), Melbourne, Australia, 2019 (JACoW, Geneva, 2019), pp. 1507-1509.

[10] X. J. Deng, R. Klein, A. W. Chao, A. Hoehl, W. H. Huang, J. Li, J. Lubeck, Y. Petenev, M. Ries, I. Seiler, C. X. Tang, and J. Feikes, Phys. Rev. Accel. Beams 23, 044001 (2020).

[11] X. J. Deng, A. W. Chao, J. Feikes, W. H. Huang, M. Ries, and C. X. Tang, Phys. Rev. Accel. Beams 23, 044002 (2020).

[12] X. Deng, A. Chao, J. Feikes, A. Hoehl, W. Huang, R. Klein, A. Kruschinski, J. Li, A. Matveenko, Y. Petenev et al., Nature (London) 590, 576 (2021).

[13] Y. Zhang, X. J. Deng, Z. L. Pan, Z. Z. LI, K. S. Zhou, W. H. Huang, R. K. Li, C. X. Tang, and A. W. Chao, Phys. Rev. Accel. Beams 24, 090701 (2021).

[14] A. W. Chao, J. Appl. Phys. 50, 595 (1979).
[15] A. W. Chao, Lectures on Accelerator Physics (World Scientific, Singapore, 2020).

[16] E. D. Courant and H. S. Snyder, Ann. Phys. (N.Y.) 3, 1 (1958).

[17] Y. Shoji, Phys. Rev. Accel. Beams 7, 090703 (2004).

[18] H. Deng and C. Feng, Phys. Rev. Lett. 111, 084801 (2013).

[19] C. Feng and Z. Zhao, Sci. Rep. 7, 4724 (2017).

[20] X. J. Deng, W. H. Huang, Z. Z. Li, and C. X. Tang, Nucl. Instrum. Methods Phys. Res., Sect. A (to be published).

[21] A. J. Dragt, F. Neri, and G. Rangarajan, Phys. Rev. A 45, 2572 (1992).

[22] J. Feikes, M. von Hartrott, M. Ries, P. Schmid, G. Wüstefeld, A. Hoehl, R. Klein, R. Müller, and G. Ulm, Phys. Rev. Accel. Beams 14, 030705 (2011).

[23] M. Ries, Ph.D. dissertation, Humboldt University of Berlin, Berlin, Germany, 2014.

[24] N. Campbell, Proc. Cambridge Philos. Soc. 15, 117 (1909).

[25] M. Sands, Phys. Rev. 97, 470 (1955).

[26] S. O. Rice, Bell Syst. Tech. J. 23, 282 (1944).

[27] J. M. Jowett, AIP Conf. Proc. 153, 864 (1987).

[28] C. Biscari, Phys. Rev. Accel. Beams 8, 091001 (2005).

[29] J. Haissinski, Nuovo Cimento Soc. Ital. Fis. B 18, 72 (1973).

[30] K. L. F. Bane, Y. Cai, and G. Stupakov, Phys. Rev. Accel. Beams 13, 104402 (2010).

[31] B. Nash, Ph.D. thesis, Stanford University, Stanford, CA, 2006.

[32] K. Kubo and K. Oide, Phys. Rev. Accel. Beams 4, 124401 (2001).

[33] A. Piwinski, in Proceedings of the 9th International Conference on High Energy Accelerators, Stanford, CA (SLAC, Stanford, 1974), p. 405.

[34] J. D. Bjorken and S. K. Mtingwa, Part. Accel. 13, 115 (1983), https://cds.cern.ch/record/140304/files/p115.pdf. 